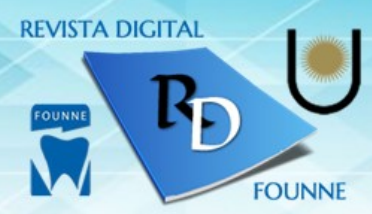

\title{
DETERMINANTES SOCIALES QUE INFLUYEN EN LA UTILIZACIÓN DEL HILO DENTAL
}

\section{Autor: María Silvina Dho}

Autor de correspondencia: msdho@odn.unne.edu.ar Av. Libertad 3400 Corrientes; Argentina

Tel: 54-3794-399579

Recepción: 8 de mayo de 2017

Aceptación: 26 de mayo de 2017

\section{RESUMEN}

Introducción: El uso del hilo dental es un método eficaz de remoción de la placa bacteriana presente en las superficies interproximales para prevenir la caries dental y la enfermedad periodontal.

Objetivo: analizar la influencia de distintos factores en la utilización diaria del hilo dental en individuos de 35 a 44 años de edad que viven en la Ciudad de Corrientes, Argentina.

Métodos: Se realizó un estudio transversal de base poblacional. La información se recolectó a través de una encuesta domiciliaria sobre conocimientos, actitudes y hábitos de salud bucal, donde figuran las variables de estudio. Se determinó el tamaño de la muestra estableciéndose un nivel de confianza del 95\%. Se entrevistó a 381 individuos. Se aplicó un diseño muestral polietápico. Se analizaron los datos con los programas SPSS 21.0 y Epidat 3.1.

*Dra. de la Universidad Nacional del Nordeste en Odontología.

Jefe de Trabajos Prácticos. Cátedra Práctica Clínica Preventiva I. Faculta de Odontología. Universidad Nacional del Nordeste. 


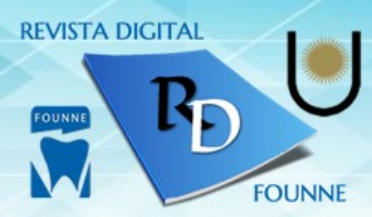

Resultados: Se halló que el $29 \%$ de los entrevistados utilizan diariamente hilo dental. El conocimiento de salud bucal; tener cobertura social y haber realizado una consulta odontológica en los últimos 12 meses anteriores a la entrevista, se asociaron significativamente al uso diario del hilo dental.

Las personas de nivel socioeconómico bajo presentaron hábitos menos adecuados de utilización. El sexo no resultó ser una variable significativa.

Conclusiones: Los programas de salud bucal, deberían contemplar los factores que influyen en la utilización diaria del hilo dental para que esta práctica se refleje en la población de estudio.

Palabras Clave: Salud Oral, Encuestas de Salud Bucal, Conductas.

\section{INTRODUCCIÓN:}

La placa bacteriana o biofilm dental es el agente causal principal de la caries y de la enfermedad periodontal; y el mejor método para prevenir los efectos nocivos de ésta son el cepillado de dientes junto con el uso de hilo dental. ${ }^{1,2}$

El control de placa interproximal es una parte importante del autocuidado oral y los pacientes deben completar el cepillado dental con técnicas de remoción interproximal de placa. Sin embargo, la realidad es que la higiene interdental no es una práctica común. Aunque los cepillos dentales han mejorado el control de la placa en las áreas interproximales, no alcanzan de forma eficiente estas superficies. En estas áreas con mucha frecuencia asientan gingivitis y periodontitis $\mathrm{y}$, además, las superficies interproximales en pacientes mayores son zonas de mayor riesgo de caries. $^{3}$

Numerosos estudios han demostrado que las prácticas de higiene bucal, tales como el cepillado dental y la utilización del hilo dental son métodos muy efectivos para controlar la placa bacteriana dentaria y reducir la incidencia de ambas patologías. ${ }^{4,5}$ En tal sentido la Asociación Dental Americana ${ }^{6}$ entre sus recomendaciones menciona el cepillado dental y la limpieza entre los dientes diariamente con hilo dental.

Un trabajo realizado en la ciudad de Corrientes documenta la alta frecuencia de utilización del cepillo dental para la higiene bucal, sin embargo, es escaso el número de personas que reportan la utilización diaria del hilo dental para completar su higiene. ${ }^{7}$ 


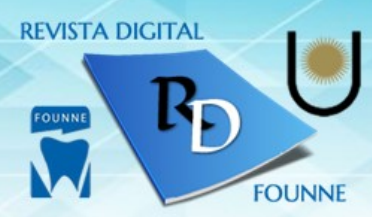

Este estudio se propone analizar la influencia de distintos factores: sexo, cobertura social odontológica, consulta odontológica, nivel socioeconómico, conocimientos de salud bucodental, en la utilización diaria del hilo dental.

\section{METODOS:}

Diseño del estudio: Se realizó un estudio descriptivo transversal de base poblacional en la Ciudad de Corrientes, capital de la provincia de Corrientes, Argentina. La metodología consistió en aplicación del método de encuestas. El relevamiento de los datos fue realizado en el año 2013 por entrevistadores instruidos en la técnica de observación directa "cara a cara".

Población de interés: La unidad de análisis estuvo compuesta por sujetos de 35 a 44 años de edad. Se focalizó en este grupo de edad porque la Organización Mundial de la Salud establece esa franja etaria como el grupo estándar de vigilancia del estado de salud bucodental de los adultos. $^{8}$

Criterios de selección: Se incluyeron en el estudio los individuos que aceptaron voluntariamente contestar las preguntas del formulario y se excluyeron los que no presentaban capacidades físicas y/o mentales para responder y con patologías que impidieran el auto-cuidado bucodental.

Estudio piloto: Se realizó una prueba piloto a 50 sujetos con características similares a la población de estudio para comprobar la adecuación del cuestionario como instrumento de medición para lograr los objetivos del estudio. Esto permitió identificar la necesidad de reformular algunas preguntas del cuestionario y simplificar su redacción.

Calculo del tamaño muestal: A partir de los datos publicados por el Instituto Nacional de Estadística y Censo (INDEC, Censo Nacional de Población, Hogares y Viviendas 2010, Provincia de Corrientes, departamento Capital) se determinó un universo de 42.242 sujetos compuesto por 19.639 hombres y 22.603 mujeres.

El tamaño de la muestra se determinó con un nivel de confianza del 95\% y un margen de error del 5\%. Para el cálculo del tamaño muestral se consideró el supuesto de máxima heterogeneidad de la muestra $50 \%$. La muestra quedó conformada por 381 sujetos $(53,5 \%$ mujeres y $46,5 \%$ hombres). 


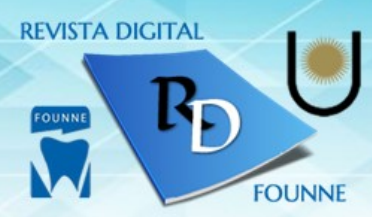

Diseño de la muestra: Se aplicó un diseño muestral aleatorio simple para la selección de las viviendas a encuestar, que se complementó con un muestreo no probabilístico por cuotas para la selección de los individuos a entrevistar, en función de la variable sexo en el rango de edad seleccionado (35 a 44 años), de acuerdo a la matriz de cuotas que se le proporcionó a cada encuestador.

\section{Variables de estudio. Medición.}

"Sexo": variable dicotómica, se consideró femenino; masculino.

"Cobertura social odontológica": esta variable hace referencia a la cobertura de salud (obra social, sistema privado de medicina prepaga, adhesión a programas y planes estatales), cuyo beneficio se extiende a la atención odontológica. La variable comprendió la siguiente pregunta: “¿Ud. presenta cobertura social odontológica?” y las posibles opciones de respuestas: “si”- “no”.

"Consulta odontológica": Se indagó por la consulta odontológica en los últimos 12 meses y por los motivos de la consulta. Esta variable comprendió dos preguntas indicadoras: “¿Asistió al odontólogo en los últimos 12 meses?” " “¿Por qué motivos asiste generalmente al odontólogo?”. “Utilización del hilo dental”: se preguntó: “¿Ud. utiliza diariamente hilo dental?”. Las posibles opciones de respuestas consideradas fueron: "si"- "no".

"Conocimientos de salud bucal": se consideraron específicamente los conocimientos referentes al hilo dental. Esta variable comprendió tres preguntas indicadoras: “¿La utilización del hilo dental, puede ayudar a prevenir la caries dental?”; “La utilización del hilo dental, puede ayudar a prevenir la inflamación de las encías?” y “Sabe cómo se utiliza el hilo dental?”. Las posibles opciones de respuestas consideradas fueron: "si"- "no".

"Nivel Socioeconómico" (NSE): para establecer el NSE de los entrevistados se utilizó un índice que comprende dos indicadores: el nivel educativo y la categoría ocupacional ${ }^{7}$ y se categorizó en bajo ( 2 a 3 puntos), medio-bajo (4 a 5 puntos), medio-medio (6 a 7 puntos), medio-alto (8 a 9 puntos) y alto (10 puntos). Atento a la escasa población de este último, para el análisis estadístico se consideraron juntos los NSE medio-alto/ alto.

Análisis de los datos: Se utilizaron los programas estadísticos SPSS 21.0 y Epidat 3.1. Se calcularon frecuencias absolutas y relativas. Para estudiar la relación entre las variables conocimientos referentes al hilo dental, sexo, cobertura social odontológica y consulta 


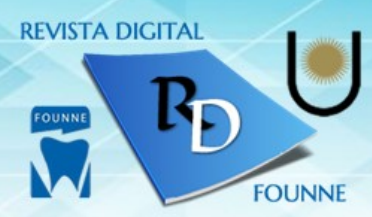

odontológica con la utilización del hilo dental se evaluó la asociación estadística mediante el odds ratio (OR) y la significación estadística con la prueba del $\mathrm{x}^{2}$, se tomó como nivel de significación aceptable un valor de 0,05. Igual nivel se utilizó para la estimación de parámetros mediante intervalo de confianza.

Para evaluar las diferencias según el NSE en la utilización del hilo dental se utilizó la prueba de comparaciones de rangos, específicamente la prueba Kruskall-Wallis, empleándose pruebas U de Mann-Whitney para valorar las diferencias de a pares. Para ello, la utilización diaria del hilo dental se consideró un hábito adecuado ( 1 punto), y la no utilización, un hábito no adecuado ( 0 punto).

Consideraciones bioéticas: Se solicitó a los sujetos su conformidad para participar del estudio, después de explicarles los objetivos del mismo y se garantizó el anonimato y confidencialidad de las respuestas. El estudio cuenta con el aval del Comité de Ética de Investigación de la Facultad de Odontología de la Universidad Nacional del Nordeste (Expediente No 04533).

\section{RESULTADOS}

La mayoría de los participantes considera que el uso del hilo dental puede ayudar a prevenir la caries dental y la inflamación gingival, pero menos de un tercio lo utilizan diariamente para completar su higiene bucal. El análisis descriptivo de las variables de estudio se muestran en la Tabla 1 .

El análisis de cada uno de los factores estudiados en función de la utilización diaria del hilo dental mostró resultados significativos para las variables: conocimiento sobre la utilización del hilo dental, reconocimiento de la utilización del hilo dental como método para prevenir la caries dental y la inflamación gingival, consulta odontológica en los últimos 12 meses y tener cobertura social odontológica (Tabla 2).

Al comparar la utilización diaria del hilo dental en los grupos conformados por distintos niveles socioeconómicos, se puede apreciar que las personas de NSE bajo muestran hábitos menos adecuados que el resto de los grupos de estudio (Figura 1), en tal sentido la prueba de comparación de rangos muestra que las personas de NSE bajo presentan puntuaciones menores que las personas de NSE medio-bajo ( $U$ de Mann-Withney $=3500, p<.013)$, medio-medio $(U$ de 


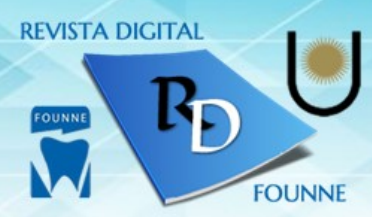

Mann-Withney $=3116, p<.001)$ y medio-alto/alto $(U$ de Mann-Withney $=1178, p<.037)$ (Tabla 3). Las comparaciones restantes no resultaron estadísticamente significativas.

\section{DISCUSIÓN}

Los resultados del estudio indican que existe una discrepancia entre el conocimiento y la práctica, pues si bien el $80,1 \%$ de los individuos declararon conocer cómo se utiliza el hilo dental, solo el 29,4\% afirmó utilizarlo diariamente.

Estos resultados concuerdan a los hallados en un estudio previo realizado en la ciudad de Corrientes ${ }^{7}$ que reportó que el $60 \%$ de encuestados manifestaron conocer cómo se utiliza el hilo dental, pero solo el 16\% tenían el hábito de utilizarlo diariamente para completar su higiene bucal. En tal sentido, múltiples investigaciones han reportado la escasa utilización del hilo dental en diferentes contextos poblacionales. ${ }^{10,11}$

La resistencia al uso del hilo dental podría deberse a la dificultad de su uso por la habilidad exigida, y la necesidad de entrenamiento, además de la pereza o falta de tiempo en adoptarlo como recurso de rutina.

Por otro lado, un mayor conocimiento referente al hilo dental se asoció significativamente a su mayor utilización. Al respecto se halló, que los individuos que tienen el conocimiento sobre el método de utilización del hilo dental, presentan 5,1 veces mayores probabilidades de utilizarlo diariamente, y los individuos que tienen el conocimiento de que la utilización del hilo dental puede ayudar a prevenir la caries dental y la inflamación gingival, presentan 4,5 y 4,6 veces respectivamente, mayores probabilidades de utilizarlo diariamente. Al respecto, hay estudios que documentan la importancia de la implementación de estrategias educativas para lograr mayor nivel de conocimientos que permitan motivar a los individuos a realizar acciones de autocuidado y de esta manera mejorar la higiene bucodental. ${ }^{12,13}$

Por otro lado, en la presente investigación se halló que la consulta odontológica en los últimos 12 meses de realizada la entrevista se asoció a una mayor probabilidad de utilización diaria del hilo dental. Los individuos que realizaron una consulta odontológica en los últimos 12 meses y aquellos que consultan generalmente por motivos preventivos, presentan 3 veces mayores probabilidades de utilizar diariamente el hilo dental. Estos resultados podrían deberse al hecho 


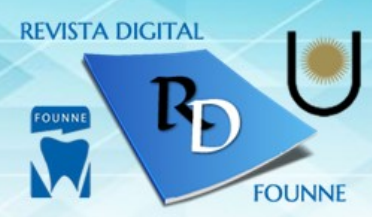

de que aquellos individuos que realizan consultas odontológicas en forma periódica, presentan mejores actitudes y predisposición hacia el autocuidado bucodental; además la consulta odontológica, propicia un ámbito en el cual podrían recibir asesoramiento profesional para mejorar sus prácticas de higiene oral.

FRIAS y col. ${ }^{14}$ reportaron en su investigación que aquellos individuos que habían consultado al odontólogo hace dos o más años presentaron menor utilización diaria del hilo dental.

En relación al nivel socioeconómico se halló que las personas de NSE bajo mostraron hábitos menos adecuados de utilización diaria del hilo dental que el resto de los grupos de estudio. Estos hallazgos podrían señalar la existencia de inequidades en el acceso a productos de higiene bucal, en particular del hilo dental.

Por otro lado, cabe señalar que, un bajo nivel socioeconómico asociado a hábitos deficientes de higiene bucal, fueron reportados como factores de riesgo de caries dental. ${ }^{15,16}$

Con respecto a la cobertura social odontológica, se halló que poco más de la mitad de la población cuenta con algún tipo de cobertura de salud. Aquellos individuos que tienen cobertura social presentan 1,6 veces más probabilidades de utilizar diariamente el hilo dental que aquellos individuos que no presentan cobertura social. Estos resultados podrían deberse a que los individuos que tienen cobertura social odontológica tienen mayor acceso a los servicios de salud odontológicos y por lo tanto mayor probabilidad de recibir atención preventiva y adiestramiento sobre el uso del hilo dental.

En relación a la variable sexo, no se halló una asociación estadísticamente significativa con el hábito de utilización diaria del hilo dental, a discrepancia de otros autores que hallaron que las personas de sexo femenino presentaron mejores hábitos de higiene oral. ${ }^{14}$, Esto podría indicar que en la población de estudio no hay mayor interés por parte de hombres y mujeres en relación a la utilización del hilo dental para completar su higiene bucal.

Limitaciones del estudio: La información recogida en la encuesta es autoreferida de forma indirecta por un informante, lo que puede introducir un sesgo de memoria y/o de deseabilidad social. Por otro lado, al tratarse de un diseño transversal, no es posible asumir causalidad en las asociaciones observadas. Igualmente, se ha logrado identificar relaciones entre las variables de estudio. 


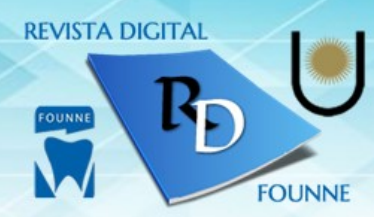

\section{CONCLUSION}

En atención a estos resultados los ejes de la implementación de programas de promoción de salud y de prevención de las enfermedades bucodentales, deberían focalizarse en buscar estrategias para que los conocimientos relacionados a la utilización del hilo dental se reflejen en la práctica de su utilización y contemplar la posibilidad de distribuir gratuitamente en forma regular y continua productos para la higiene oral, especialmente a los sectores más desfavorecidos de la población, acompañado con las instrucciones necesarias para una correcta higiene oral.

\section{REFERENCIAS}

1. Gómez Arcila V, Verbel Bohórquez J., Díaz Caballero A., Arroyo Salgado B. Enfoque hacia la dinámica de la biopelícula oral para el control de enfermedades bucales prevalentes. Rev. Clínica de Medicina de Familia. 2014;7(2):153-155.

2. CUENCA SALA E, BACA GARCÍA P. Odontología Preventiva y Comunitaria. Principio, métodos y aplicaciones. $4^{\circ}$ ed. Elseiver, España, 2013.

3. LÓPEZ CASTELlaNOS G, LOPEZ RAMIREZ LB, NACHON GARCIA MG, HERNANDEZ LUNAGOMEZ D. Salud bucal, salud bucal percibida y calidad de vida en adultos mayores. Rev Med UV. [Internet]. 2014; [citado 04 mayo 2017];6-11. Disponible en: http://www.medigraphic.com/pdfs/veracruzana/muv-2014/muv142a.pdf

4. LÓPEZ SOTO, CEREZO CORREA M. "EFECTIVIDAD Y PREFERENCIA DE LA RAFIA COMO MATERIAL DE LIMPIEZA DENTAL INTERPROXIMAL”. UNIVERSITAS ODONTOLÓGICA [INTERNET]. 2011; [CITADO 04 MAYO 2017]; DISPONIBLE EN:

FILE://D:/USUARIO/DOWNLOADS/DIALNETEFECTIVIDADYPREFERENCIADELARAF IACOMOMATERIALDELIMP-3670221.PDF

5. GONZÁlEZ RIVAS BE, RAMÍREZ PÉREZ EK, HERRERA HUIZA MB, MATTÍA REINOZA MG, MEZA OM, RAMÍREZ Y. Efectividad de los tratamientos para el control de la placa dental. Revisión Sistemática. Rev Venez Invest Odont IADR 2016; 4(2): 330-952. 


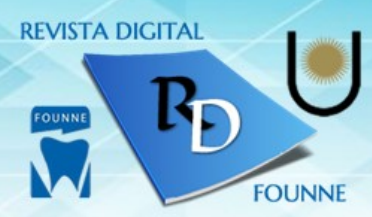

6. AMÉRICA DENTAL ASOCIATTION. Hábitos saludables. [Internet]. [citado 04 mayo 2017]; Disponible en: http://www.mouthhealthy.org/en/adults-under-40/healthy-habits/.

7. DHÓ MS, VILA VG, PALLADINO AC. "Situación de salud bucal de pacientes mayores de 18 años. Cátedra Práctica Clínica Preventiva I, Facultad de Odontología UNNE, Argentina, 2010”. Rev. Fac Odontol. Univ. Antioq. 2013; 24(2): 214-31.

8. ORGANIZACIÓN MUNDIAL DE LA SALUD. Oral Health Surveys. Basic Methods. $5^{\text {th }}$ Edition. 2013

9. DAL-BÓ CORADINI MC, PERES MA. "Determinantes da utilização de serviços odontológicos entre adultos: um estudo de base populacional em Florianópolis, Santa Catarina, Brasil”. Cad. Saúde Pública. 2013; 29 (11): 2319-32.

10. LÓPEZ AP, OSPINA D, HERRERA Y, BETANCUR J, AGUDELO. HIGIENE BUCAL EN LA POBLACIÓN DE SAN FRANCISCO ANTIOQUIA Y SUS FACTORES RELACIONADOS. REV. NAC. DE ODNTOL. UNIVERSIDAD COOPERATIVA DE COLOMBIA. 2016; 12 (22): 23-30

11. MARTÍNEZ-MEJÍA VJ, QUIROGA GARCÍA MA, MARTÍNEZ GONZÁLEZ GI, AGUIAR FUENTES EG. Determinantes sociales y percepción de salud bucal de las gestantes del Hospital Civil de TEPIC. Rev. de Salud Pública y Nutrición. 2017; 16(1): 16-22

12. MATOS CANTILLO DM Y COL. Intervención educativa sobre salud bucal en adolescentes venezolanos. Rev Inf Cient. 2017; 96(3):70-78

13. INOCENTE-DÍAZ ME, PACHAS-BARRIONUEVO F. Educación para la Salud en Odontología. Revista Estomatológica Herediana. 2012; 22(4): 232-41

14. FRIAS AC, MYSUGUTI AH, SANCHEZ TP, MANTOVANI GL, PESTANA SR. Social determinants and use of dental floss by 35-44-year-old adults. RGO. 2012; 60 (3): 321-27.

15. COSTA S, MARTINS C, BONFIM M, ZINA L, PAIVA S, PORDEUS I y col. Systematic Review of Socioeconomic Indicators and Dental Caries in Adults. Int. J. Environ. Res. Public Health. 2012; 9(10): 3540-74.

16. ÁlVAREZ L, LIBERMAN J, ABREU S, MANGARELli C, CORREA MB., DEMARCO FF. et al. "Dental caries in Uruguayan adults and elders: findings from the first Uruguayan National Oral Health Survey”. Cad. Saúde Pública. 2015; 31 (8):1663-72. 


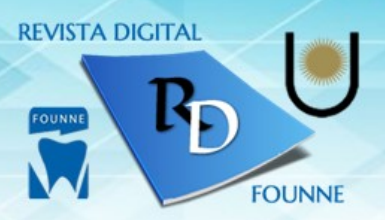

Tabla 1: Análisis descriptivo de las variables de estudio. En porcentajes ( $n=381)$. Individuos de 35 a 44 años. Ciudad de Corrientes; Argentina. 2013.

\begin{tabular}{|lr} 
Sexo & \\
Femenino & 53,5 \\
Masculino & 46,5 \\
& \\
Nivel Socioeconómico & 1,6 \\
Alto & 10,8 \\
Medio-alto & 35,2 \\
Medio-medio & 36,7 \\
Medio-bajo & 15,7 \\
Bajo & \\
Cobertura social odontológica & \\
Con cobertura & 56,4 \\
Sin cobertura & 43,6 \\
Consulta Odontológica en los últimos 12 meses posteriores a la entrevista & \\
Individuos que consultaron & 67,2 \\
Individuos que no consultaron & 32,8 \\
& \\
Motivos de la consulta & 27,0 \\
Control/ chequeo dental & 34,6 \\
Tratamientos dentales & 38,6 \\
Dolor/ Otras urgencias & \\
Conocimientos de salud bucodental & \\
La utilización diaria del hilo dental puede ayudar a prevenir la caries dental & 73,8 \\
La utilización diaria del hilo dental puede ayudar a prevenir la inflamación gingival & 68,2 \\
Conocimiento sobre la utilización del hilo dental & 80,1 \\
& \\
Uso diario del hilo dental & \\
Individuos que utilizan diariamente & 29,4 \\
Individuos que no utilizan diariamente & 70,6 \\
\hline & \\
\hline
\end{tabular}

Tabla 2: Factores asociados a la utilización diaria del hilo dental.

Individuos de 35 a 44 años. Ciudad de Corrientes; Argentina. 2013. 


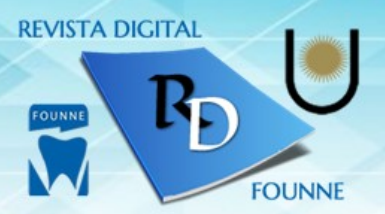

\begin{tabular}{|c|c|c|c|}
\hline Variables & OR & IC $95 \%$ & Valor de $p$ \\
\hline Sexo & 0,96 & $0,62-1,50$ & 0,88 \\
\hline Cobertura social odontológica & 1,67 & $1,06-2,64$ & 0,02 \\
\hline Consulta odontológica en los últimos 12 meses & 3,18 & $1,83-5,52$ & 0,00 \\
\hline Consulta al odontólogo por motivos de control/chequeo dental & 3,09 & $1,92-4,99$ & 0,00 \\
\hline $\begin{array}{l}\text { Conocimiento de que la utilización del hilo dental puede ayudar a } \\
\text { prevenir la caries dental }\end{array}$ & 4,53 & $2,31-8,89$ & 0,00 \\
\hline $\begin{array}{l}\text { Conocimiento de que la utilización del hilo dental puede ayudar a } \\
\text { prevenir la inflamación gingival }\end{array}$ & 4,62 & $2,50-8,51$ & 0,00 \\
\hline Conocimiento de cómo debe utilizarse el hilo dental & 5,17 & $2,29-11,66$ & 0,00 \\
\hline
\end{tabular}

Figura 1: Utilización del hilo dental en relación al Nivel socioeconómico

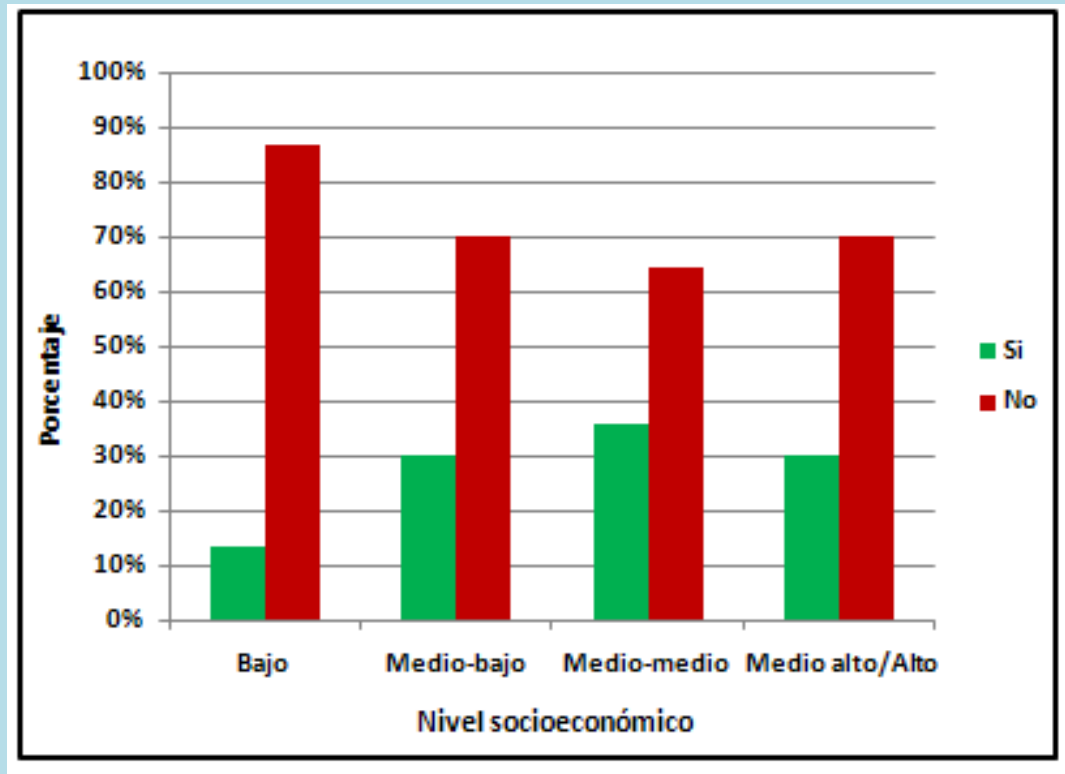




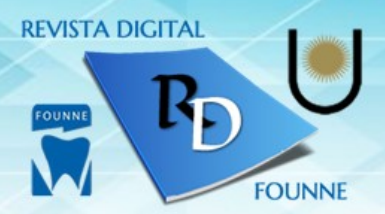

Tabla 3.

Rangos promedios y pruebas no paramétricas para la utilización diaria dental según el NSE

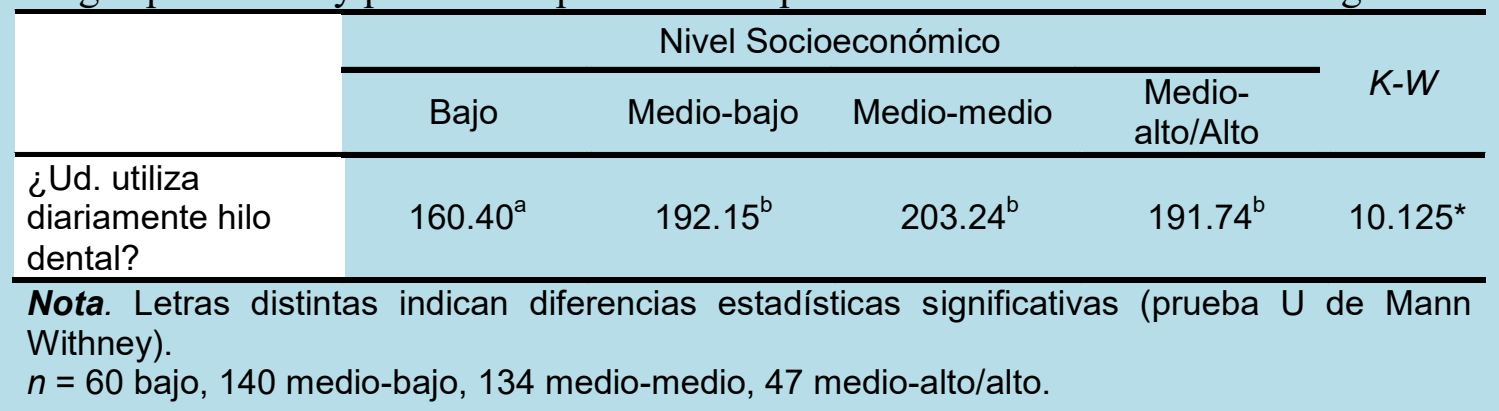

